\title{
LINKS WITH UNLINKING NUMBER ONE ARE PRIME
}

\author{
C. MCA. GORDON AND J. LUECKE
}

(Communicated by Ronald Stern)

ABSTRACT. We prove that a link with unlinking number one is prime.

\section{INTRODUCTION}

Let $L$ be a link in $S^{3}$. The unlinking number of $L, u(L)$, is defined to be the least number of times $L$ must pass through itself iri order to transform it into the unlink. It is not hard to see that this is the same as the minimum, over all diagrams of $L$, of the least number of crossing changes in that diagram needed to transform $L$ to the unlink.

Recall that a connected sum of links $L_{1}$ and $L_{2}$ is any link $L$ obtained by removing the interior of a trivial ball-pair $\left(B_{i}^{3}, B_{i}^{1}\right) \subset\left(S^{3}, L_{i}\right), i=1,2$, and then gluing the resulting pairs along their boundaries $\left(S_{i}^{2}, S_{i}^{0}\right)$. It is convenient to write $L=L_{1} \# L_{2}$, although in general $L$ is not uniquely determined by $L_{1}$ and $L_{2}$.

A natural conjecture, which is certainly classical at least in the case of knots, is the following.

Conjecture. $u\left(L_{1} \# L_{2}\right)=u\left(L_{1}\right)+u\left(L_{2}\right)$.

We shall say that $L$ is prime if $L=L_{1} \# L_{2}$ implies that $L_{1}$ or $L_{2}$ is an unlink. (Note that although this is the definition of primality that is appropriate in our present context, it may not be the usual one; it differs from that given in [KT], for example.) Since $u(L)=0$ if and only if $L$ is an unlink, the above Conjecture implies that if $u(L)=1$ then $L$ is prime. In the case of knots, this was proved by Scharlemann [S]. Here we prove the analog for links with more than one component.

Theorem. Let $L$ be a link with more than one component such that $u(L)=1$. Then $L$ is prime.

Received by the editors July 23, 1992.

1991 Mathematics Subject Classification. Primary 57M25.

Key words and phrases. Unlinking number, prime.

The first author was partially supported by NSF grant NSF-DMS-9001478. The second author was partially supported by NSF grants NSF-DMS-8903599, NSF-DMS-9158090 and a Sloan Foundation Fellowship. 
An alternative proof of Scharlemann's theorem was given by Zhang $[Z]$, by applying the main result of [GL1] to the 2-fold branched cover of the knot. The present proof follows the same philosophy, using [GL2] instead of [GL1].

\section{PROOF}

Let $K$ be a knot in the interior of an orientable 3-manifold $M$. Let $N(K)$ be a tubular neighborhood of $K$, and let $\alpha$ be the isotopy class of an essential simple closed curve in $\partial N(K)$. The manifold obtained by $\alpha$-Dehn surgery on $K$, which we will denote by $K(\alpha)$, is the result of attaching a solid torus $V$ to $M$ - int $N(K)$ by identifying $\partial V$ with $\partial N(K)$ in such a way that $\alpha$ bounds a disk in $V$. If $\alpha$ and $\beta$ are two such isotopy classes in $\partial N(K)$ then $\Delta(\alpha, \beta)$ denotes their minimal geometric intersection number.

If $L$ is a link in $S^{3}$, let $M(L)$ denote the 2-fold branched cover of $L$, with branched covering projection $p: M(L) \rightarrow S^{3}$ and canonical involution $h: M(L) \rightarrow M(L)$.

Lemma 1. Let $L, L^{\prime}$ be links in $S^{3}$ such that $L^{\prime}$ is obtained from $L$ by a single crossing change. Then there exists a knot $K$ in $M(L)$ such that

(i) $K$ has an h-invariant tubular neighborhood $N(K)$ such that $p(N(K))$ is a 3-ball that meets $L$ in an unknotted pair of arcs;

(ii) $M\left(L^{\prime}\right)$ is homeomorphic to $K(\alpha)$ for some $\alpha$ with $\Delta(\alpha, \mu)=2$, where $\mu$ is the meridian of $K$.

Proof. This follows from [L, Proof of Lemma 1]. (See also [M].)

Note that if $L$ is the $n$-component unlink, $n \geq 1$, then

$$
M(L) \cong \underset{n-1}{\#} S^{1} \times S^{2}
$$

The next lemma gives the converse.

Lemma 2. Let $L$ be a link in $S^{3}$ such that $M(L)$ is homeomorphic to $\#_{n-1} S^{1} \times S^{2}$. Then $L$ is the $n$-component unlink.

Proof. The case $n=1$ is the $\mathbb{Z}_{2}$-Smith Conjecture [W]. The general case follows easily by induction on $n$ using [KT, Lemma 1].

Finally, we shall need the following fact about Dehn surgeries that yield reducible manifolds.

Lemma 3. Let $K$ be a knot in a 3-manifold $M$ such that $M-K$ is irreducible but $K(\alpha)$ and $K(\beta)$ are reducible. Then

$$
\Delta(\alpha, \beta) \leq 1 \text {. }
$$

Proof. This is [GL2, Corollary 1.2].

Proof of Theorem. Let $L$ be an $n$-component link in $S^{3}, n \geq 2$, with $u(L)=$ 1. First note that if $L$ is a split union $L_{1} \sqcup L_{2}$, then clearly it must be that $L_{1}$ (say) is an unlink and $u\left(L_{2}\right)=1$. Hence we may assume that $L$ is nonsplit and, therefore, that $S^{3}-L$ is irreducible. 
Suppose for contradiction that $L$ is a connected sum $L_{1} \# L_{2}$, where $L_{i}$ is not an unlink, $i=1,2$. Then $M(L) \cong M\left(L_{1}\right) \# M\left(L_{2}\right)$. In particular, since $L_{i}$ is not the unknot, $i=1,2, M(L)$ is reducible, by the $\mathbb{Z}_{2}$-Smith Conjecture [W].

Since $u(L)=1$, we can apply Lemma 1 with $L^{\prime}$ the $n$-component unlink, giving a knot $K$ in $M(L)$, with meridian $\mu$, such that

$$
K(\alpha) \cong M\left(L^{\prime}\right) \cong \underset{n-1}{\#} S^{1} \times S^{2}
$$

where $\Delta(\alpha, \mu)=2$. But $K(\mu) \cong M(L)$ is also reducible. It follows from Lemma 3 that $X=M(L)$ - int $N(K)$ is reducible.

Choosing $N(K)$ as in part (i) of Lemma 1, let $B_{0}$ be the 3-ball $S^{3}-$ int $p(N(K))$, and let $L_{0}=L \cap B_{0}$. Then $X$ is the 2-fold branched cover of $\left(B_{0}, L_{0}\right)$. Since $h$ restricts to an involution on $X$, [KT, Lemma 1] implies that $X$ contains an essential 2-sphere $S$ (one that does not bound a 3-ball) such that either $h(S) \cap S=\varnothing$ or $h(S)=S$ and $S$ meets $p^{-1}(L)$ transversely.

In the first case, $p(S)$ is a 2-sphere in $B_{0}-L_{0}$. Since $S^{3}-L$ is irreducible by assumption, $p(S)$ bounds a 3-ball $B$ in $B_{0}-L_{0}$. But then $B$ lifts to a 3-ball in $X$ bounded by $S$, contradicting the essentiality of $S$.

In the second case, $S$ must meet $p^{-1}(L)$ in two points. Then $p(S)$ is a 2-sphere in $B_{0}$ meeting $L_{0}$ in two points. Let $B_{1}$ be the 3-ball in $B_{0}$ bounded by $p(S)$, and let $L_{1}=L \cap B_{1}$. Then in $X, S$ bounds $M_{1}$, the 2 -fold branched cover of $\left(B_{1}, L_{1}\right)$. Since $M_{1} \subset X \subset M\left(L^{\prime}\right) \cong \#_{n-1} S^{1} \times S^{2}, M_{1}$ is homeomorphic to $\#_{k-1} S^{1} \times S^{2}$ minus the interior of a 3-ball, where $k \leq n$. By Lemma 2 and the assumption that $L$ is nonsplit, it follows that $L_{1}$ is an unknotted arc in $B_{1}$, giving $M_{1} \cong B^{3}$ and again contradicting the essentiality of $S$.

\section{ADDED IN PROOF}

After this paper was accepted for publication we learned that the theorem of the title is contained in Theorem 2 of [E-M]. So our paper should be regarded as giving a new proof of Eudave-Muñoz's result, in which the explicit combinatorial arguments of [E-M] are replaced by a reference to the main result of [GL2] (whose proof is based on more complicated combinatorial arguments). In the same way, our approach gives alternative proofs of Theorems 1, 2, and 3 of [E$\mathrm{M}$ ]. Eudave-Muñoz was also aware of the fact that the primality of unknotting number one knots follows from [GL1] (see [E-M, p. 775]).

\section{REFERENCES}

[E-M] M. Eudave-Muñoz, Primeness and sums of tangles, Trans. Amer. Math. Soc. 306 (1988), 773-790.

[GL1] C. McA. Gordon and J. Luecke, Only integral Dehn surgeries can yield reducible manifolds, Math. Proc. Cambridge Philos. Soc. 102 (1987), 97-101.

[GL2] _ Reducible manifolds and Dehn surgery (to appear).

[KT] P. K. Kim and J. L. Tollefson, Splitting the PL involutions of nonprime 3-manifolds, Michigan Math. J. 27 (1980), 259-274.

[L] W. B. R. Lickorish, The unknotting number of a classical knot, Contemp. Math., vol. 44, Amer. Math. Soc., Providence, RI, 1985, pp. 117-121.

[M] J. M. Montesinos, Surgery on links and double branched coverings of $S^{3}$, Ann. of Math. Stud., no. 84, Princeton Univ. Press, Princeton, NJ , 1975, pp. 227-259. 
[S] M. G. Scharlemann, Unknotting number one knots are prime, Invent. Math. 82 (1985), 37-55.

[W] F. Waldhausen, Über Involutionen der 3-Sphäre, Topology 8 (1969), 81-91.

[Z] X. Zhang, Unknotting number one knots are prime: a new proof, Proc. Amer. Math. Soc. 113 (1991), 611-612.

Department of Mathematics, The University of TeXas at Austin, Austin, TeXas 787121082

E-mail address: gordon@math utexas .edu

E-mail address: luecke@math .utexas .edu 